\title{
The Outcome of Open Surgical Treatment of Posttraumatic Elbow Stiffness
}

\author{
Mohamed Othman*, Ahmed El-Malt, Ahmed Nahla \\ Department of Orthopedics, Faculty of Medicine, Zagazig University, Egypt. \\ yousufmmkh@gmail.com
}

\begin{abstract}
Background: Posttraumatic stiff elbow is a frequent and disabling complication and causes difficulty in placement of hand in space and hence limits the functional capacity. It poses challenges for management. The initial management should be nonoperative and surgical treatment is indicated when conservative measures for a reasonable period fail to achieve the functional range of motion. The aim of this study was to assess the outcome of open arthrolysis of posttraumatic elbow stiffness.

Patients and Methods: A prospective study was conducted at our institution, between 2013 and 2016, on open surgical release of posttraumatic elbow stiffness in 16 elbows of 16 patients (who were available for follow-up examination). The mean age of the patients was 22 \pm 4.2 years (range: $12-36$ yrs). The mean time interval between injury and surgical release was $8.3 \pm 2$ months (range: $3-12$ months). The surgical approach was: lateral $(n=10)$, medial $(n=3)$ and combined lateral and medial $(n=3)$. All patients underwent extensive rehabilitation, starting immediately after the procedure.

Results: The mean follow-up period was $8.5 \pm 2$ months (range: 7 -13months), The preoperative total flexion/ extension arc improved from a mean of $40^{\circ} \pm 10.5$ to $105^{\circ} \pm 21.5$ (60 to 135) at the final follow-up, with a mean gain of $65^{\circ} \pm 4$. The mean preoperative Mayo Clinic Elbow Performance Score of $60 \pm 17.5$ points (20-70), improved at the final follow-up to $85 \pm 8.8$ points ( 70 to 100 ), with a mean gain of $25 \pm 4.5$ points. The results were excellent in six elbows, good in seven, fair in two and poor in one. Therefore, a satisfactory functional outcome was achieved in $81.25 \%$ of cases. Superficial infection occurred in one patient (that cured on antibiotic and local care) but, no deep infection. One case of preoperative ulnar nerve deficit recovered finally after anterior transposition. Two patients had postoperative transient paresthesia of ulnar nerve, but without a measurable motor or sensory deficit. Recurrence of stiffness occurred in one patient (6.25\%), who was a 12 years old uncooperative patient.
\end{abstract}

Conclusion: The open release of posttraumatic elbow stiffness provides a useful gain in range of motion and marked improvement of elbow function, especially in motivated co-operative patients.

Keywords: Mayo Clinic Performance Score; Open Arthrolysis; Post-Traumatic Stiff Elbow

\section{INTRODUCTION}

Post-traumatic elbow stiffness is a common, but little discussed clinical problem [1]. The presence of three articulations with a single synovial tissue-lined capsule, the close proximity of the joint capsule to the ligaments and muscles, and the intrinsic congruity of the humeroulnar articulation as well as the frequency of comminuted fractures in this region and even the prolonged immobility used by some orthopedists have all been suggested as predisposing factors [2,3]. Significant elbow stiffness has both functional and cosmetic limitations. It may cause severe functional impairment because of the inability to position the hand in space [1-3]. Morrey et al [3] considered that the minimum functional range of motion (ROM) necessary for carrying out activities of daily 
living was an arc between $130^{\circ}$ of flexion and $-30^{\circ}$ of extension, with $50^{\circ}$ of both pronation and supination, thus totaling a range of $100^{\circ}$ both in the sagittal and in the coronal plane. Posttraumatic contractures can be classified into extrinsic (extra-articular), intrinsic (intra-articular) or mixed pathology [4]. The initial treatment of post-traumatic stiffness of the elbow, particularly when it is extrinsic, should be non-operative. This includes the use of splints( dynamic or static) and physiotherapy. Surgical treatment is indicated when conservative management fails to achieve the minimal functional ROM.[1,4,6]. Surgical release can be performed using a lateral, a medial, combined lateral and medial, an anterior or a posterior approach [5-9]. The aim of this study was to evaluate the outcome of open surgical release of posttraumatic elbow stiffness.

\section{Patients And Methods}

A prospective study was conducted at our institution, between 2013 and 2016, on open surgical release of posttraumatic elbow stiffness in 16 elbows of 16 patients (who were available for follow-up examination). Surgery was indicated when there was a persistent significant elbow contracture, causing functional impairment [flexion contracture $>30^{\circ}\left(>30^{\circ}\right.$ extension loss $)$ and/or flexion range is $<130^{\circ}$ ], despite conservative management including physiotherapy for an enough period. This was at least 6 months, but was only 3 months if there was a hard mechanical block to movement (bony or mispositioned hardware) [9-11]. The exclusion criteria were nontraumatic causes, inadequate soft-tissue envelope, or burn, unmotivated noncooperative patients and noncompliant patients, such as those with uncontrolled psychiatric conditions or substance abuse. Ten patients were male and six were female, with a mean age of $22 \pm 4.2$ years (range: $12-36$ ys.). The dominant limb was affected in nine patients. The study was approved by Institutional Ethical Committee and a written consent to participate in the study after explanation of risks and benefits, was taken.

Preoperative assessment included history taking and examination of both elbows and of shoulder and hand. Clinical assessment included measurement of ROM by goniometry and functional assessment by Mayo Elbow Performance Score (MEPS) [12]. Imaging included plain X-rays and CT with 3D reformats. Photographs and videos were done for all cases. EMG/NCV were done if there was nerve deficit. An important step was discussion with the patients as regards realistic expectations and rehabilitation program [8]. The primary injury was intercondylar fracture $(n=3)$, elbow dislocation $(n=2)$, radial head fracture $(n=2)$, fracture of capitellum $(n=1)$, fracture of medial condyle $(n=1)$, fracture-dislocation $(n=4)$ and local trauma without fracture $(n=3)$. Their treatments included ORIF, closed reduction /casting and splinting. (Figure 1).

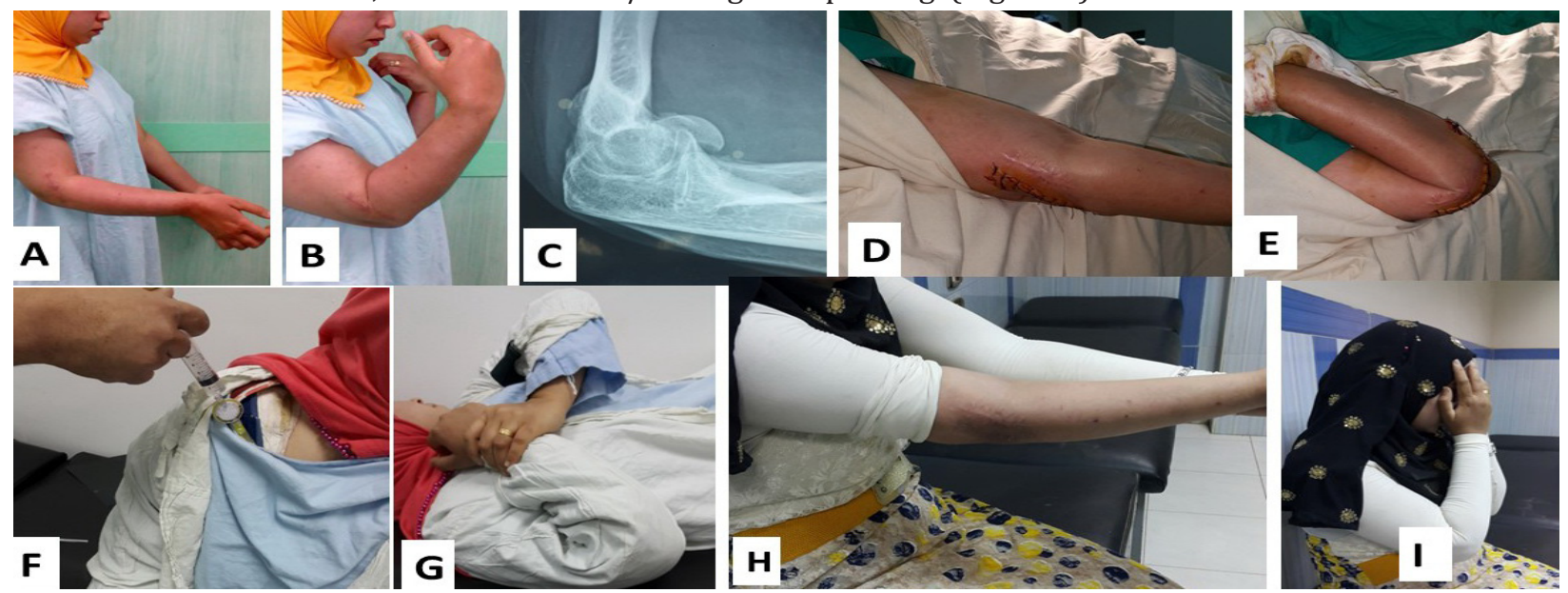

Fig1. A, B, C) A thirty years old lady with a posttraumatic Rt elbow stiffness of 10 months-duration, after failed ORIF of capitellum fracture. The preoperative ROM = about 30ㅇ (60-90); D, E) Intraoperative correction \& ROM; $F, G)$ Painless physiotherapy under a regional brachial plexus block; H, I) Final postoperative ROM=about $80^{\circ}$ (20-100), with a gain of about $50^{\circ}$. 


\section{OPERATIVE Procedures}

Anesthesia was ultrasonic-guided supraclavicular brachial plexus block with insertion of an indwelling catheter to allow prolonged postoperative pain control and painless early mobilization [13,14]. General anesthesia was added in irritable patients $(n=5)$. Surgery was done in the supine position, without tourniquet. The surgical approach was: lateral $(n=10)$, medial $(n=3)$ and combined lateral and medial $(n=3)$. The medial approach was indicated for preoperative ulnar nerve deficit $(n=1)$ and removal of implants $(n=2)$. Combined lateral and medial approaches were used for severe flexion contractures. Whatever be the approach, to improve elbow flexion, remove posterior soft tissue tethers (posterior joint capsule and triceps muscle adhesions) and anterior mechanical blocks to flexion (fibrous tissue, hardware or osteophytes on coronoid process or in coronoid and radial fossae). To improve elbow extension, posterior impingement at the olecranon tip and floor of olecranon fossa (fibrous tissue, osteophytes, loose bodies or hardware) is removed, and the anterior tethering capsule and brachialis are released [8] (Figure 2).

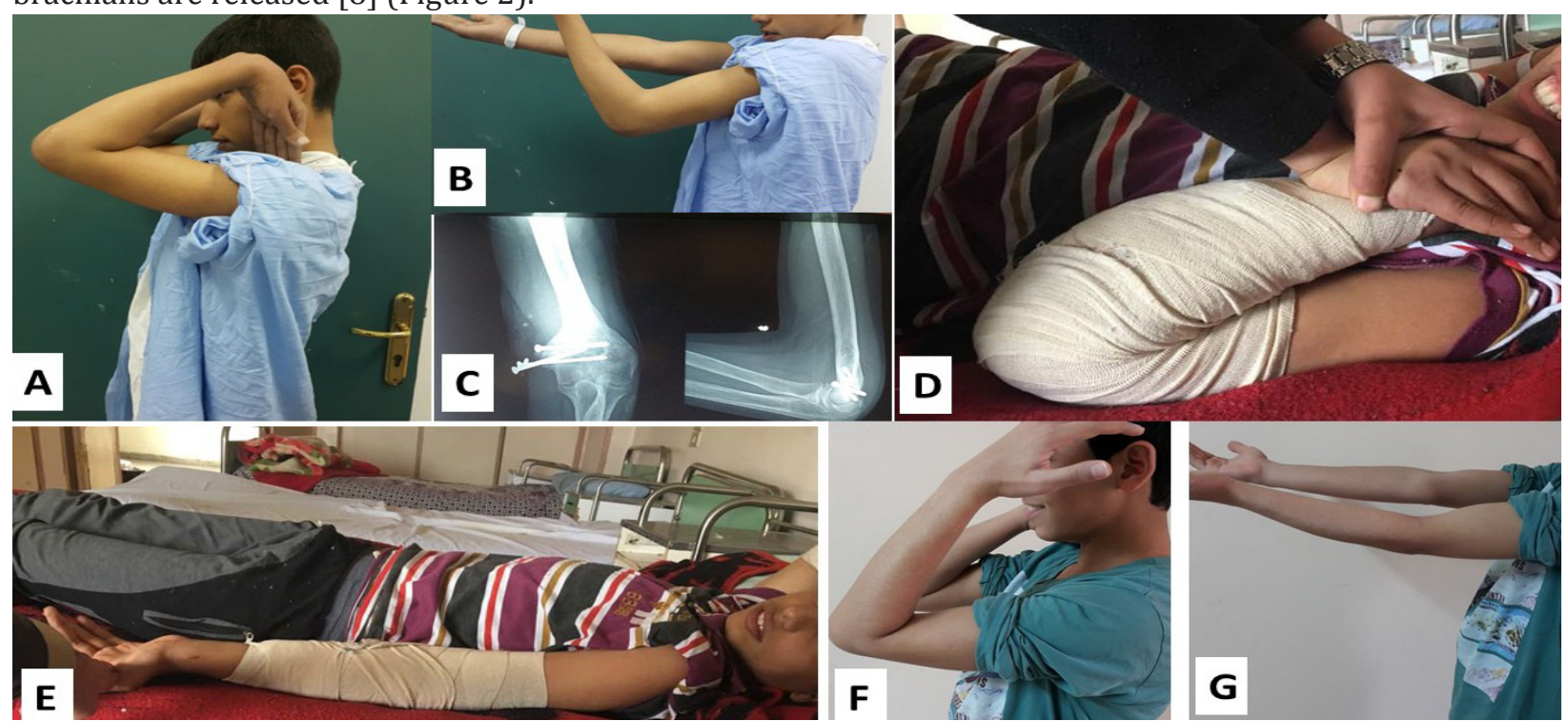

Fig2. A, B, C) A fourteen years old patient with a Lt elbow stiffness and ulnar nerve deficit after ORIF of medial condyle fracture. The preoperative ROM was about 45 (75-120); D, E) Postoperative painless ROM under a regional brachial plexus block; F, G) Final outcome: ROM=100 (15-115), with a gain of 55․

Every effort is made to preserve the lateral collateral ligament and the anterior band of the medial collateral ligament (MCL), to maintain the stability of the joint $[4,5,10]$.

(a) Lateral column approach - proximal to the elbow joint, this approach is between the humerus and ECRL anteriorly and the humerus and triceps posteriorly. Distal to the joint, this approach is between ECRL and ECRB. Posteriorly, the capsule is incised, and olecranon and olecranon fossa are approached. Anteriorly, the muscle mass is taken off the capsule, which is then excised to get access to the anterior compartment. The view toward the medial side is limited through this incision, and if any procedure is required on the medial side, a separate incision on the medial side is required [5,15-17].

(b) Medial column approach - the ulnar nerve is isolated and mobilized. Posteriorly, the posterior band of MCL is cut and excised to improve flexion beyond $100^{\circ}$. The triceps is reflected off the humerus, and posterior elbow capsule is cut, thus reaching the posterior pathology. Anteriorly, the brachialis is raised off the humerus after cutting the medial septum. The anterior half of the flexor-pronator muscle mass is raised from the medial epicondyle in continuation with the distal brachialis. The medial anterior capsule is excised and any anterior pathology is dealt with. $[8,18,19]$. 
Finally, flexion and extension of the elbow were carried out passively to evaluate the gain in movement. Hemostasis was obtained. After surgery, the wound was closed with suction drainage. A long-arm splint was applied with the elbow in extension or flexion depending on the direction in which movement was more resistant to pas manipulation, or in $90^{\circ}$ of flexion when there was no major direction of limitation, as proposed by Park et al. [1].

Other procedures that were used in association included: removal of synthesis material in 6 cases, excision of the radial head $(n=2)$, excision of ununited part of capitellum $(n=1)$, resection of the tip of the olecranon $(n=8)$, ulnar nerve simple release without transposition $(n=1)$ and anterior transposition of the ulnar nerve $(n=5)$. This was done for preoperative complaint of paresthesia and when tension in the nerve following joint release was noted.

\section{Postoperative Management}

Physiotherapy was started on the $2^{\text {nd }}$. postoperative day. To allow this, pain control was achieved with injection of $0.25 \%$ marcaine through the supraclavicular indwelling catheter, three times daily. This produced complete sensory block with maintenance of some motor power $[13,14]$. We started with passive mobilization then passive and active-assisted full ROM. Between physiotherapy sessions, a splint was worn. The patients were kept hospitalized for two weeks (time of removal of stitches and of indwelling catheter). The patients then were discharged and instructed sharply to do physiotherapy (passive, active-assisted and active elbow movements to full range) under supervision of a therapist and at home. At night, the patient wore a splint with the elbow in the position of maximum flexion or extension which could be obtained without excessive pain. Three weeks after surgery, the splint was totally discarded and the patients were encouraged to do vigorous active movements and to use their elbows as much as possible through a full ROM during the day to help maintain the motion that was achieved at operation. The patients were followed-up by a coordinated therapist daily for the first six weeks. The physical therapy was continued for at least 3 months or till a plateau was reached. The patients were followed-up by us weekly for the first three months, then monthly thereafter. The supportive therapy was given for the first six weeks. It included control of swelling (by elevation, ice and NSAIDs), tubular compression bandage (for elbow support and control of swelling) and a fixed dose of $25 \mathrm{mg}$ of Indomethacin twice daily for a period of six weeks (to prevent heterotopic ossification) [8].

\section{Statistical analysis}

A statistical analysis was performed with SPSS version 15.0 (SPSS, Chicago, Illinois). Means and standard deviations were calculated. The paired $t$ test was used for comparison of preoperative and postoperative values. Significance was set at P-value $<0.05$.

\section{RESULTS}

The mean follow-up period was $8.5 \pm 2$ months (range: 7 - 13 months). The mean pre-operative limitation of extension of $40^{\circ} \pm 10.5(20-85)$ was reduced by the final follow-up to a mean of $20^{\circ} \pm 5.5$ (5 to 30 ) and the mean flexion increased from $80^{\circ} \pm 15.8$ (45-125) to $125^{\circ} \pm 7.5$ (100 to 135). The preoperative total flexion/ extension arc improved from a mean of $40^{\circ} \pm 10.5$ to $105^{\circ} \pm 21.5$ (60 to 135) at the final follow-up, with a mean gain of $65^{\circ} \pm 4$. The mean pre-operative pronation improved from $50^{\circ} \pm 15.4$ (10 to 80) to $65^{\circ} \pm 17.3$ (20 to 90 ) and supination increased from $55^{\circ} \pm 18.6$ (10 to 90 ) to $70^{\circ} \pm 16.9$ (20 to 90 ). The mean preoperative Mayo Clinic Elbow Performance Score of $60 \pm 17.5$ points (20-70), improved at the final follow-up to $85 \pm 8.8$ points (70 to 100 ), with a mean gain of $25 \pm 4.5$ points. The results were excellent in 6 elbows, good in 7 , fair in two and poor in one. Therefore, a satisfactory functional outcome was achieved in $81.25 \%$ of cases. All changes was found to be statistically- significant $(\mathrm{P}<0.001)$. (Table 1$)$. 
The Outcome of Open Surgical Treatment of Posttraumatic Elbow Stiffness

Table1. The Mayo Clinic Elbow Performance score [12]. Overall score: Excellent :( 90 - 100 points), Good: (75 - 89 points), Fair: (60 - 74 points) and Poor: (<60 points) [12]. ADLs = daily living activities.

\begin{tabular}{|c|c|c|}
\hline Variable & Definition & Points \\
\hline \multirow{4}{*}{ Pain $(\max =45 \mathrm{p})$} & No & 45 \\
\hline & Mild & 30 \\
\hline & Moderate & 15 \\
\hline & Severe & 0 \\
\hline \multirow{3}{*}{ Arc of motion $(\max =20 \mathrm{p})$} & $>100^{\circ}$ & 20 \\
\hline & $50-100^{\circ}$ & 15 \\
\hline & $<50^{\circ}$ - & 5 \\
\hline \multirow{3}{*}{ Stability $(\max =10 \mathrm{p})$} & Stable & 10 \\
\hline & Mild instability & 5 \\
\hline & Gross instability & 0 \\
\hline \multirow{5}{*}{$\begin{array}{l}\text { Function; } 5 \text { ADLs } \\
(\max =25 \mathrm{p})\end{array}$} & Able to perform personal hygiene & 5 \\
\hline & Able to comb hair & 5 \\
\hline & Able to feed himself & 5 \\
\hline & Able to put on shirt & 5 \\
\hline & Able to put on shoes & 5 \\
\hline Total & & 100 points \\
\hline
\end{tabular}

\section{Complications}

Superficial infection occurred in one patient (that cured on antibiotic and local care) but, no deep infection. One case of preoperative ulnar nerve deficit recovered finally after anterior transposition. Two patients had postoperative transient paresthesia of ulnar nerve, but without a measurable motor or sensory deficit. Recurrence of stiffness occurred in one patient (6.25\%). This was a 12 ys. old patient who did not cooperate and complete the rehabilitation program. We did manipulation under anesthesia with improvement of ROM, but he again did not cooperate and finally rated poor. No case of heterotopic ossification had occured.

\section{Discussion}

Elbow stiffness is a frequent and disabling complication of elbow trauma [9-11]. Surgical release is indicated in posttraumatic stiff elbows when non-operative treatment has failed and function is severely impaired [1-5]. Patient selection is a very critical point, as not all patients are suitable candidates for surgical release. Before planning the procedure, the surgeon should try to assess the expectations of the patient and the limitations that the patient is facing and exclude unmotivated noncooperative patients, as the success of treatment is dependent on patient's understanding and willingness to comply with a rigorous postoperative protocol $[6,8,10]$.

Timing of elbow arthrolysis is controversial. Many authors advised that nonoperative treatment should be continued for at least six months before operative treatment [1,2,4,10,20]. By contrast, Swaroop et al, [19] suggested a shorter waiting time before surgical treatment (for a shorter rehabilitation time, an earlier return to work and a better prognosis) and he proposed surgery after an initial active physiotherapy for three months of trauma, if it failed to achieve the minimal functional ROM. In our study, we operated after at least 3-6 months to allow for the inflammatory phase of soft tissue healing to resolve, as proposed by many authors $[4,8,9]$.

A useful arc of about $100^{\circ}$ with a painless stable joint is the objective $[4,6]$.

In our series, the total flexion/extension arc improved from a mean of $40^{\circ}$ to $105^{\circ}$, with a mean gain of $65^{\circ}$. The mean preoperative Mayo Elbow Performance score of 60 points, improved at the final follow-up to 85 points, with a mean gain of 25 points. The results were excellent in 6 elbows, good in 7, fair in two and poor in one. Therefore, a satisfactory functional outcome occurred in $81.25 \%$ of cases. Our results compare favorably with previous reports that have shown an overall improvement in movement of the elbow [1,2,4,6,7,11,16,17,20-22].

American Research Journal of Orthopedics and Traumatology

Page 5 


\section{The Outcome of Open Surgical Treatment of Posttraumatic Elbow Stiffness}

Different surgical approaches have been described. The choice of approach is based on surgeon preference and patient factors such as the site of any previous incision, the presence of neuropathy, the location of periarticular ossifications, intra-articular deformities and mispositioned hardware. The surgical approach chosen must allow all relevant pathology to be addressed [1,16,18,21-25]. The proponents of the lateral approach are its simplicity, less wound problems and direct access to both the anterior and posterior ulnohumeral and radiocapitellar joints through one incision, with preservation of the collateral ligaments. Its opponents are (a) inability to safely reach the medial side to treat cases with ulnar neuropathy or calcifications in MCL. (b) Ulnar paresthesia is the main complication seen after the release by lateral incision. This was attributed to traction neuritis caused by the abrupt increase in elbow flexion during the operation [2,5,16,17]. The advantages of the medial approach are (a) direct access to the ulnar nerve (for safe release and/or transposition), the posterior oblique bundle of MCL (proposed to be a restraint to terminal elbow flexion) and anterior and posterior aspects of the elbow joint, via one medial incision, thus providing subsequent safe access to the anterior and posterior aspects of the elbow. (b) a more favorable cosmetic scar. The disadvantages are (a) inability to address lateral joint pathology (b) proximity of the medial antebrachial cutaneous nerve (c) potentially greater muscle morbidity [6-8,18]. In most instances, a complete elbow release can be performed through either approach, but occasionally a dual (medial and lateral) approach is required to address all relevant pathology [24]. However, in patients requiring a combined medial and lateral approach, a single posterior skin incision has been advocated to address both the medial and lateral aspects of the elbow and to lower the incidence of cutaneous nerve injuries that can occur with more direct medial and lateral approaches. However,this incision requires raising large skin flaps that may lead to subsequent seroma formation [1,24,25]. Urbaniak et al. [25] described an anterior approach. It was used for escision of an extensive anterior heterotopic ossification. However, it does not allow addressing any posterior pathology [26].

The most frequently reported complications after open elbow release are ulnar neuritis, wound complications (including wound hematoma and infection), loss of ROM, heterotopic ossification, pain, complex regional pain syndrome, triceps insufficiency, and instability $[6,10,11,16,17,19-27]$. The significant complications in our series were transient ulnar paresthesia in two and recurrence of stiffness in one patient. The ulnar nerve deserves special mention. Ulnar nerve decompression ( \pm anterior transposition) through a medial approach is indicated for preoperative nerve symptoms, in severe flexion contractures and for cases of iatrogenic ulnar nerve deficits $[1,5,6,8,10,18,24]$.

Although there is no consensus in the literature for the optimum postoperative rehabilitation protocol, there is agreement that postoperative physiotherapy plays an equally important role to surgery itself in the final outcome and it should be instituted as early as possible, to prevent intra-articular adhesion formation. It is very important in minimizing the loss of movement gained at operation, which is mandatory in all cases $[1,2,10,23,24,27]$. The success of treatment is dependent on patient's understanding and willingness to comply with a rigorous postoperative protocol $[7,8,19]$. Our policy was to keep the patients hospitalized for two weeks to start and supervise painless physiotherapy under the effect of a regional brachial plexus block. We did not use the continuous passive motion machine (CPM). After discharge from the hospital, physiotherapy was continued for at least three months or till ROM became stabilized. Mittal [6] proposed keeping the patients hospitalized for a few days to start and then supervise the physiotherapy of the operated elbow and for a better pain control. Streubel and Cohen [8] proposed that prolonged in-hospital CPM with the use of an indwelling regional block catheter may be warranted in cases of severe contractures. The use of CPM is controversial. Many authors reported the use of CPM for variable periods in their postoperative rehabilitation program $[2,8,25]$. Others did not use it with a comparable outcome $[1,6,10,11]$. The duration of physiotherapy is variable in the literature. It varies from three months up to 12 months or till a plateau in ROM is reached $[6,11]$.

Although manipulation under anesthesia have been recommended for the management of the stiff elbow, it may be most indicated for recurrent elbow stiffness after surgical release. Reported complications with this

American Research Journal of Orthopedics and Traumatology

Page 6 
procedure include transient ulnar neuritis and the potential for ligament disruption and fracture $[8,28,29]$. Arthroscopic arthrolysis has become a current practice for treating post-traumatic stiff elbow, with consistent results. However, this is a technically complex procedure for surgeons. It should preferably be indicated in cases of less severe contracture ( $\mathrm{ROM}>80^{\circ}$ and extension deficit $<40^{\circ}$ ), with no significant bone lesions to be resected $[30,31]$. Hinged elbow fixator, is recommended when collaterals are disrupted during the contracture release [6].

\section{CONCLUSIONS}

Elbow stiffness is a frequent and disabling complication after trauma to the elbow. Surgical release is indicated if conservative treatment failed to achieve the functional range of movement. Correct patient selection, clear understanding of the pathological anatomy, careful attention to surgical technique with preservation of the elbow stabilizers, and structured postoperative rehabilitation program are mandatory to achieve a sustained optimal outcome.

\section{REFERENCES}

1. Park MJ, Kim HG, Lee JY (2004) Surgical treatment of post-traumatic stiffness of the elbow. J Bone Joint Surg Br 86: 1158-1162.

2. Gundlach U and Eygendaal D (2008) Surgical treatment of posttraumatic stiffness of the elbow: 2-year outcome in 21 patients after a column procedure. Acta Orthop 79: 74-77.

3. Morrey BF, Askew LJ, Chao EY (1981) A biomechanical study of normal functional elbow motion. J Bone Joint Surg 63-A: 872-877.

4. Morrey BF (2005) Posttraumatic Stiff Elbow: Clinical orthopedics and related research 431: 26-35.

5. Cohen MS and Hastings H (1998) Posttraumatic contracture of the elbow: operative release using a lateral collateral ligament sparing approach. J Bone Joint Surg 80-B: 805-812.

6. Mittal R (2017) Posttraumatic stiff elbow. Indian J Orthop 51: 4-13.

7. Filho GM and Galvão MV (2010) Post-traumatic stiffness of the elbow. Rev Bras Ortop 45: 347-356.

8. Streubel PN and Cohen MS (2015) Open Surgical Release for Contractures of the Elbow. J Am Acad Orthop Surg 23: 328-338.

9. Stans AA, Maritz NGJ, O’Driscoll SW, Morrey BF (2002) Operative treatment of elbow contracture in patients twenty-one years of age or younger. J Bone Joint Surg Am 84: 382-387.

10. Rex C, Kumar S, Srimannarayana A, Chugh S, Ravichandran M, et al. (2008) Analysis of results of surgical treatment of posttraumatic stiff elbow. Indian J Orthop 42: 192-200.

11. Miyazaki AN, Fregoneze M, Santos PD, da Silva LA, Di Giunta G, et al. (2010) Evaluation of the results of surgical treatment of posttraumatic stiffness of the elbow in skeletally mature patients. Rev Bras Ortop 45: 529-537.

12. Morrey BF and An KN (2000) In: Morrey BF, editor. The elbow and its Disorders: Functional evaluation of the elbow. 3rd ed. Philadelphia: WB Saunders 2000.

13. Duggan E, El Beheiry H, Perlas A, Lupu M, Nuica A, et al. (2009) Minimum effective volume of local anesthetic for ultrasound-guided supraclavicular brachial plexus block.Reg Anesth Pain Med 34: 215-218.

14. Gupta PK and Hopkins PM (2013) Effect of concentration of local anaesthetic solution on the $\mathrm{ED}_{50}$ of bupivacaine for supraclavicular brachial plexus block. Br J Anaesth 111: 293-296.

15. Husband JB and Hastings $H$ (1990) The lateral approach for operative release of posttraumatic contracture of the elbow. J Bone Joint Surg Am 72: 1353-1358. 
16. Mansat $P$ and Morrey BF (1998) The column procedure: A limited lateral approach for extrinsic contracture of the elbow. J Bone Joint Surg Am 80: 1603-1615.

17. Cohen MS (2007) Open capsular release for soft tissue contracture of the elbow, In Yamaguchi K, King GJW, McKee M, et al, eds: Advanced Reconstruction: Elbow. Rosemont, IL, American Academy of Orthopaedic Surgeons 2007: 195-204.

18. Wada T, Ishii S, Usui M, Miyano S (2000) The medial approach for operative release of post-traumatic contracture of the elbow. J Bone Joint Surg Br 82: 68-73.

19. A Swaroop, S Avasthi, M Yadav, S Kumar, A Bharti, et al. (2009) Assessment of Results of Open Arthrolysis. In Post Traumatic Stiff Elbows. The Internet Journal of Orthopedic Surgery 11.

20. Tan V, Daluiski A, Simic P, Hotchkiss RN (2006) Outcome of open release for post-traumatic elbow stiffness. J Trauma;61(3):673-8.

21. Charalambous CP, Morrey BF (2012) Posttraumatic elbow stiffness. J Bone Joint Surg Am.;94(15):1428-37

22. Kodde IF, van Rijn J, van den Bekerom MP, Eygendaal D (2013) Surgical treatment of post-traumatic elbow stiffness: a systematic review. J Shoulder Elbow Surg.;22(4):574-80.

23. Mellema JJ, Lindenhovius A LC, Jupiter JB (2016) The posttraumatic stiff elbow: an update. Curr Rev Musculoskelet Med. Jun; 9(2): 190-198.

24. Tosun B, Gundes H, Buluc L, Sarla AY (2007) The use of combined lateral and medial releases in the treatment of post-traumatic contracture of the elbow. International Orthopaedics 31: 635-638.

25. Bręborowicz M, Lubiatowski P, Długosz J, Ogrodowicz P, Wojtaszek M, et al. (2014) The outcome of open elbow arthrolysis: comparison of four different approaches based on one hundred cases. International Orthopaedics 38: 561-567.

26. Urbaniak JR, Hansen PE, Beissinger SF, Aitken MS (1985) Correction of post-traumatic flexion contracture of the elbow by anterior capsulectomy. J Bone Joint Surg Am 67: 1160-1164.

27. Sharma S and Rymaszewski LA (2007) Open arthrolysis for post-traumatic stiffness of the elbow: Results are durable over the medium term.J Bone Joint Surg Br 89: 778-781.

28. Duke JB, Tessler RH, Dell PC (1991) Manipulation of the stiff elbow with patient under anesthesia. J Hand Surg. 1991;16(1):19-24.

29. Araghi A, Celli A, Adams R, Morrey B (2010) The outcome of examination (manipulation) under anesthesia on the stiff elbow after surgical contracture release. J Shoulder Elbow Surg 19: 202-208.

30. Ball CM, Meunier M, Galatz LM, Calfee R, Yamaguchi K (2002) Arthroscopic treatment of stiff elbow. J Shoulder Elbow Surg 11(6): 624-629.

31. Wu X, Wang H, Meng C, Yang S, Duan D, Xu W, et al.(2015) Outcomes of arthroscopic arthrolysis for the post-traumatic elbow stiffness. Knee Surg Sports Traumatol Arthrosc.;23(9):2715-20.

Citation: Mohamed Othman, Ahmed El-Malt, Ahmed Nahla. "The Outcome of Open Surgical Treatment of Posttraumatic Elbow Stiffness". American Research Journal of Orthopedics and Traumatology. 2017; 2(1): 1-8.

Copyright (C) 2017 Mohamed Othman, Ahmed El-Malt, Ahmed Nahla. This is an open access article distributed under the Creative Commons Attribution License, which permits unrestricted use, distribution, and reproduction in any medium, provided the original work is properly cited. 